\title{
Identification of two cancer-associated fibroblast markers revealing stromal heterogeneity in sustaining cancer progression and chemoresistance
}

\author{
Alexia Brunel, Rémi Samain, Cindy Neuzillet, Corinne Bousquet \\ INSERM U1037, Cancer Research Center of Toulouse-University Toulouse III Paul Sabatier, Toulouse, France \\ Correspondence to: Corinne Bousquet. INSERM UMR-1037, Centre de Recherches en Cancérologie de Toulouse (CRCT), 2 avenue Hubert Curien, \\ Oncopôle Entrée C, CS53717, 31037 Toulouse cedex1, France. Email: corinne.bousquet@inserm.fr. \\ Comment on: Su S, Chen J, Yao H, et al. CD10(+)GPR77(+) Cancer-Associated Fibroblasts Promote Cancer Formation and Chemoresistance by \\ Sustaining Cancer Stemness. Cell 2018;172:841-56.e16.
}

Submitted May 19, 2018. Accepted for publication Jun 07, 2018.

doi: 10.21037/tcr.2018.06.09

View this article at: http://dx.doi.org/10.21037/tcr.2018.06.09

Intra-tumor cellular heterogeneity is a key player of tumor aggressiveness and resistance to therapies. Genetic diversity in tumor cells enables, under selective pressures (e.g., during the metastatic process or under therapies), the rise of certain subclones proficient with survival and metastatic advantages (1). Yet, phenotypic tumor cell heterogeneity also critically dictates such adaptative behaviours, this plasticity relying on stromal signals that reversibly change signaling pathways or gene expression programs in tumor cells, and reciprocally $(2,3)$. An illustration of the importance of this phenotypic heterogeneity in tumor cells and crosstalks with the stroma has been revealed in pancreatic cancer, the aggressiveness of which is closely linked to transcriptomic and epigenetic signatures $(4,5)$. Tumor stroma is represented by acellular (extracellular matrices, vesicles, soluble proteins and lipids) and cellular components [cancer-associated fibroblasts (CAF), endothelial cells, immune cells and nerve cells], the variety of which underscores the complexity of tumor biology. Tumor stroma heterogeneity also ensues from its constant evolution during tumor progression or remodeling under treatments, and from inconsistent spatial distribution into the tumor of stromal components (6-8). Consequently, tumor cells will not, at a specific stage of tumor progression, be exposed to the same signals whether they localize within the primary tumor or at metastatic site, in a small or a large lesion, and in the central area or at the invasive fronts, underlying the great advances that should in a near future bring multi-spatial single cell phenotyping approaches. Another step of diversity within the stroma concerns the immune system which comprises, even within a similar immune cell class, not only antitumoral, but also pro-tumoral, cell subsets (e.g., M1 vs. M2 macrophages populations), these populations being evolving to functionally divergent subsets owing to tumor- or stromal (particularly, CAF)-derived polarizing factors (9-11). This implies that, depending on the factors released by the whole tumor, an anti- or pro-tumoral immunity will be built up, this signature impacting on patient prognosis and response to therapy (12). For example, it is now recognized that a stromal anti-tumoral lymphocyte T CD8 signature is of good prognosis in many cancers $(13,14)$, albeit not sufficient since CD8 cytolytic activity is modulated by other microenvironment factors (myeloid cells, cytokines, hypoxia) (15).

Interestingly, Su et al. recently reported that different CAF subsets may also exist, dramatically impacting on prognosis (16). Indeed, these authors demonstrated that the presence, in breast and lung tumors, of a specific subset of CAFs identified with two novel CAF cell-surface markers (CD10 and GPR77), is predictive of cancer patient response to neoadjuvant therapy and outcome. Authors describe that only CAFs positive for CD10 and GPR77 provide chemoresistance to tumor cells, implying that all CAF subsets don't present this feature. One can speculate that the emergence of this CAF subpopulation is triggered by the tumor biology and the selective pressure resulting 
from exposure to treatment, and $\mathrm{Su}$ et al. show that CD10and GPR77-positive CAFs are enriched in tumors from neoadjuvant-treated patients, as compared to naïve patient tumors, and are resistant in vitro to chemotherapies. Interestingly, CAFs not only express mechanisms for chemotherapeutic escape which remain to be understood, but also scavenge active drugs from pancreatic cancer cells contributing to the clinical failure of chemotherapies in this cancer (17).

Existence of heterogeneity within CAFs is recent and comes from reports in pancreatic cancer where pro- and anti-tumoral CAF subsets are suspected $(18,19)$. In this dramatic yet incurable disease, in which stroma represents up to $80 \%$ of the tumor mass, strategies aimed at cotargeting CAFs or the extracellular matrices, in addition to cancer cells, are nowadays tested in clinical trials. Clinical trials aimed at inhibiting the sonic hedgehog pathway were a dismal failure (20) and NCT01130142, although its genetic invalidation had previously showed in preclinical studies in mice promising anti-tumoral effect potentiating the cytotoxic effects of chemotherapy (21). Although this proliferative pathway is specifically activated in pancreatic CAFs, its chronic inhibition in a pancreatic cancer mouse model and in pancreatic cancer patients had opposite effect than expected, i.e., pro-tumoral consequences with increased tumor angiogenesis, tumor cell metastasis [increased epithelial-to-mesenchymal transition (EMT)] and cachexia $(19,22)$. The subsequent conclusion that CAFs may have anti-tumoral functions was also put forward by another study in which the genetic invalidation, in another preclinical transgenic mouse model of pancreatic cancer, of a specific subset of CAFs [proliferative and $\alpha$-smooth muscle actin ( $\alpha$-SMA)-positive] similarly triggered EMT in tumor cells as well as immunosuppression [increase in T-regulatory cells (Treg)] (18). The hypothesis of the existence of different CAF subsets with distinct functions was further highlighted again in pancreatic cancer where both $\alpha$-SMA-positive and -negative CAF populations were described, and also associated with different spatial tumor distribution (distance to tumor cells) and function (IL-6dependent inflammatory features) (23). Unraveling real functions of CAFs is challenged by the lack of specific CAF markers. Most commonly described markers (e.g., $\alpha$-SMA, FSP-1, FAP, PDGFR $\beta$ ) are not when taken individually specific CAF markers. Nevertheless, combination of six markers including the four previously cited has enabled to delineate in breast tumors four CAF subsets, which differently express those proteins, and one subset (subset 1) being endowed with immunosuppressive activities (24). Interestingly, each breast tumor comprises the four different CAF subsets (i.e., intra-tumoral CAF heterogeneity) but those enriched with subset 1 may develop the ability to acquire resistance to immunotherapies. One can therefore speculate that strategies aimed at specifically targeting this breast tumor CAF subset (deletion or reprogrammation) may be therapeutically promising in contrast to invalidating the three other subsets although their functions are unknown. The hypothesis of whether certain CAF subsets are anti-tumoral remains to be demonstrated. One can't exclude that unexpected pro-tumoral effects resulting in pancreatic cancer from anti-CAF therapeutic strategies may have be generated through adaptative mechanisms in surrounding tumor and/or other stromal cells, in a similar manner as those observed in tumors treated with antiangiogenic therapies whereby the resulting hypoxia triggers a tumor cell aggressive phenotype (25). CAF normalization rather than complete elimination seems more appropriate to avoid deleterious adaptative effects. Secretory features of CAFs including of the cytokine IL- 6 and the chemokine CXCL-12 are associated with pro-tumoral inflammatory and immunosuppressive features, respectively $(16,24)$. CAF secretory activity was reported to be significantly normalized by targeting protein synthesis, hence described to be increased in pancreatic CAFs as compared to nonactivated fibroblasts (26). In the article of Su et al., the most aggressive CAF subset (CD10- and GPR77positive) is shown to secrete IL- 6 and IL- 8 providing a cancer survival stem cell niche, facilitating patient-derived xenograft engraftment in mice (Figure 1). This IL-6 and IL-8 paracrine pathway was described to be sustained in the CD10 and GPR77-positive CAF subset by an autocrine loop involving the complement signaling onto the C5a GPR77 receptor, which triggers transcriptional activation through the NFkB pathway of IL-6 and IL-8 promoters. Interestingly, blocking this pathway using a GPR77neutralizing antibody abolished tumor aggressiveness and chemoresistance induced by the CD10 and GPR77positive CAF subset. Emerging promoting roles in cancer progression were described for complement anaphylatoxins including $\mathrm{C} 5 \mathrm{a}$, which was reported to promote chronic inflammation, immunosuppression, angiogenesis, and cancer cell metastatic potential (27), but the GPR77C5a autocrine loop had previously not been functionally highlighted in CAFs. In contrast, stromal expression of the cell membrane-associated metalloproteinase CD10 was reported as a prognostic marker in malignancies and 


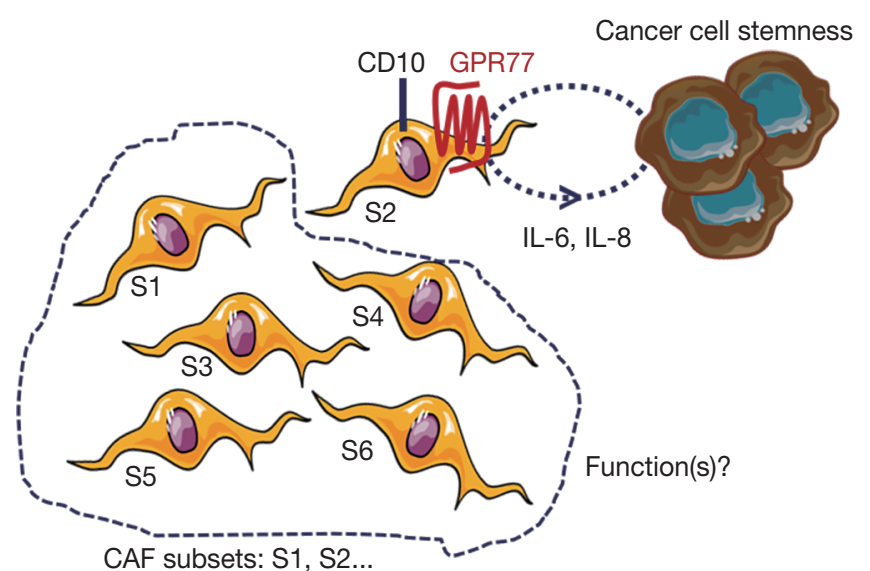

Figure 1 A specific subset of CAFs expressing the both markers CD10 and GPR77 is able to sustain cancer cell stemness and chemoresistance. This relies in this CAF subset on a complement GPR77-mediated autocrine loop resulting in IL-6 and IL-8 secretion. Whether other CAF subsets (i.e., CD10 and GPR77negative) have a specific impact on the tumor biology remains to be investigated. CAF, cancer-associated fibroblasts.

associated with biologic aggressiveness, but also already in 2010 as a marker for distinguishing a pro-metastatic CAF subset (28). Whether CD10 has a functional role in CAF biology or whether is just a "passenger" CAF subset marker remains to be explored. Adding the GPR77 marker on top of CD10, Su et al. further define a specific and functionally distinct pro-tumoral subset within CAF populations, although cells expressing both markers are not guaranteed to be actual CAFs. Targeting both proteins together could help to develop more precise therapy with the goal to specifically target the pro-tumoral CAF subset, as opposed to the others which may be "good" subsets, yet to be demonstrated. Finally, whether the GPR77- and CD10-positive CAF subset alone supports all the previously described pro-tumoral CAF features, especially their dialog with other stromal cells, is also an open-question (29).

\section{Acknowledgments}

The authors would like to thank Servier Medical Art for their image bank used to create the illustrations.

Funding: CRCT team 6 is supported by LNCC (Label Ligue Nationale Contre le Cancer) and the French National Institute of Cancer (INCa, PLBIO13-134 and PLBIO15-115). R Samain and C Neuzillet salaries are funded by Fondation pour la Recherche Médicale.

\section{Footnote}

Provenance and Peer Review: This article was commissioned and reviewed by the Section Editor Chen Qian (Center for Inflammation \& Epigenetics, Houston Methodist Hospital Research Institute, Houston, USA).

Conflicts of Interest: All authors have completed the ICMJE uniform disclosure form (available at http://dx.doi. org/10.21037/tcr.2018.06.09). The authors have no conflicts of interest to declare.

Ethical Statement: The authors are accountable for all aspects of the work in ensuring that questions related to the accuracy or integrity of any part of the work are appropriately investigated and resolved.

Open Access Statement: This is an Open Access article distributed in accordance with the Creative Commons Attribution-NonCommercial-NoDerivs 4.0 International License (CC BY-NC-ND 4.0), which permits the noncommercial replication and distribution of the article with the strict proviso that no changes or edits are made and the original work is properly cited (including links to both the formal publication through the relevant DOI and the license). See: https://creativecommons.org/licenses/by-nc-nd/4.0/.

\section{References}

1. Hunter KW, Amin R, Deasy S, et al. Genetic insights into the morass of metastatic heterogeneity. Nat Rev Cancer 2018;18:211-23.

2. Schneider G, Schmidt-Supprian M, Rad R, et al. Tissuespecific tumorigenesis: context matters. Nat Rev Cancer 2017;17:239-53.

3. Tape CJ, Ling S, Dimitriadi M, et al. Oncogenic KRAS Regulates Tumor Cell Signaling via Stromal Reciprocation. Cell 2016;165:1818.

4. Bailey P, Chang DK, Nones K, et al. Genomic analyses identify molecular subtypes of pancreatic cancer. Nature 2016;531:47-52.

5. Nicolle R, Blum Y, Marisa L, et al. Pancreatic Adenocarcinoma Therapeutic Targets Revealed by Tumor-Stroma Cross-Talk Analyses in Patient-Derived Xenografts. Cell Rep 2017;21:2458-70.

6. Terry S, Buart S, Chouaib S. Hypoxic Stress-Induced Tumor and Immune Plasticity, Suppression, and Impact on Tumor Heterogeneity. Front Immunol 2017;8:1625. 
7. Barker HE, Paget JT, Khan AA, et al. The tumour microenvironment after radiotherapy: mechanisms of resistance and recurrence. Nat Rev Cancer 2015;15:409-25.

8. Knudsen ES, Vail P, Balaji U, et al. Stratification of Pancreatic Ductal Adenocarcinoma: Combinatorial Genetic, Stromal, and Immunologic Markers. Clin Cancer Res 2017;23:4429-40.

9. Spranger S, Gajewski TF. Impact of oncogenic pathways on evasion of antitumour immune responses. Nat Rev Cancer 2018;18:139-47.

10. Thommen DS, Schumacher TN. T Cell Dysfunction in Cancer. Cancer Cell 2018;33:547-62.

11. Kiss M, Van Gassen S, Movahedi K, et al. Myeloid cell heterogeneity in cancer: not a single cell alike. Cell Immunol 2018;330:188-201.

12. Mariathasan S, Turley SJ, Nickles D, et al. TGFbeta attenuates tumour response to PD-L1 blockade by contributing to exclusion of T cells. Nature 2018;554:544-8.

13. Bindea G, Mlecnik B, Tosolini M, et al. Spatiotemporal dynamics of intratumoral immune cells reveal the immune landscape in human cancer. Immunity 2013;39:782-95.

14. Carstens JL, Correa de Sampaio P, Yang D, et al. Spatial computation of intratumoral $\mathrm{T}$ cells correlates with survival of patients with pancreatic cancer. Nat Commun 2017;8:15095.

15. Tsujikawa T, Kumar S, Borkar RN, et al. Quantitative Multiplex Immunohistochemistry Reveals MyeloidInflamed Tumor-Immune Complexity Associated with Poor Prognosis. Cell Rep 2017;19:203-17.

16. Su S, Chen J, Yao H, et al. CD10(+)GPR77(+) CancerAssociated Fibroblasts Promote Cancer Formation and Chemoresistance by Sustaining Cancer Stemness. Cell 2018;172:841-56.e16.

17. Hessmann E, Patzak MS, Klein L, et al. Fibroblast drug scavenging increases intratumoural gemcitabine accumulation in murine pancreas cancer. Gut 2018;67:497-507.

18. Ozdemir BC, Pentcheva-Hoang T, Carstens JL, et al. Depletion of carcinoma-associated fibroblasts and fibrosis induces immunosuppression and accelerates pancreas

Cite this article as: Brunel A, Samain R, Neuzillet C, Bousquet C. Identification of two cancer-associated fibroblast markers revealing stromal heterogeneity in sustaining cancer progression and chemoresistance. Transl Cancer Res 2018;7(Suppl 7):S718S721. doi: 10.21037/tcr.2018.06.09 cancer with reduced survival. Cancer Cell 2014;25:719-34.

19. Rhim AD, Oberstein PE, Thomas DH, et al. Stromal elements act to restrain, rather than support, pancreatic ductal adenocarcinoma. Cancer Cell 2014;25:735-47.

20. Catenacci DV, Junttila MR, Karrison T, et al. Randomized Phase Ib/II Study of Gemcitabine Plus Placebo or Vismodegib, a Hedgehog Pathway Inhibitor, in Patients With Metastatic Pancreatic Cancer. J Clin Oncol 2015;33:4284-92.

21. Olive KP, Jacobetz MA, Davidson CJ, et al. Inhibition of Hedgehog signaling enhances delivery of chemotherapy in a mouse model of pancreatic cancer. Science 2009;324:1457-61.

22. Lee JJ, Perera RM, Wang H, et al. Stromal response to Hedgehog signaling restrains pancreatic cancer progression. Proc Natl Acad Sci U S A 2014;111:E3091-100.

23. Ohlund D, Handly-Santana A, Biffi G, et al. Distinct populations of inflammatory fibroblasts and myofibroblasts in pancreatic cancer. J Exp Med 2017;214:579-96.

24. Costa A, Kieffer Y, Scholer-Dahirel A, et al. Fibroblast Heterogeneity and Immunosuppressive Environment in Human Breast Cancer. Cancer Cell 2018;33:463-79.e10.

25. Crawford Y, Ferrara N. Tumor and stromal pathways mediating refractoriness/resistance to anti-angiogenic therapies. Trends Pharmacol Sci 2009;30:624-30.

26. Duluc C, Moatassim-Billah S, Chalabi-Dchar M, et al. Pharmacological targeting of the protein synthesis mTOR/4E-BP1 pathway in cancer-associated fibroblasts abrogates pancreatic tumour chemoresistance. EMBO Mol Med 2015;7:735-53.

27. Ajona D, Ortiz-Espinosa S, Pio R. Complement anaphylatoxins $\mathrm{C} 3 \mathrm{a}$ and $\mathrm{C} 5 \mathrm{a}$ : Emerging roles in cancer progression and treatment. Semin Cell Dev Biol 2017. [Epub ahead of print].

28. Ikenaga N, Ohuchida K, Mizumoto K, et al. CD10+ pancreatic stellate cells enhance the progression of pancreatic cancer. Gastroenterology 2010;139:1041-51, 51.e1-8.

29. LeBleu VS, Kalluri R. A peek into cancer-associated fibroblasts: origins, functions and translational impact. Dis Model Mech 2018;11. doi: 10.1242/dmm.029447. 